\title{
Wave-dissipating Performance of Twin-plate Breakwater Under Oblique Random Waves
}

\author{
Qian Gu, Guoxing Huang and Ningchuan Zhang \\ State Key Laboratory of Coastal and Offshore Engineering, Dalian University of Technology \\ Dalian, Liaoning Province, China \\ $\mathrm{Cun} \mathrm{Hu}$ \\ Institute of Mechanics, Chinese Academy of Sciences \\ Beijing, China
}

\begin{abstract}
The present study investigates experimentally the wave-dissipating performance of twin-plate breakwater under oblique random waves. Through extensive model tests, the effects of the relative plate width and relative wave height on the dissipating performance are investigated under various incident wave angles. The results show that the dissipating performance changes insignificantly within the measured range of $15^{\circ} \sim 60^{\circ}$ incident angles. The transmission coefficient $k t$ under oblique waves fluctuates around the value under normal waves. In addition, the transmission was directly affected by the relative plate width, i.e., the transmission was $0.1<k t<0.15$ for $B / L<0.5$ and $0.05<k t<0.10$ for $B / L \geq 0.5$. In particular, under oblique waves the wave patterns after the twin-plate breakwater exhibited significant three-dimensional characteristics, which were different from those under normal incident waves. To validate the present experimental results, the measured transmission coefficients are compared to those of Neelamani and Gayathri (2006) under normal incident waves, and the comparison shows good agreement.
\end{abstract}

\section{INTRODUCTION}

Conventional breakwaters have been widely used in coastal engineering all over the world. However, the cost for the construction of conventional breakwaters increases exponentially with the increase in the water depth. Furthermore, the conventional breakwaters obstruct natural currents, which could lead to hydroenvironmental issues. The permeable breakwater, on the one hand, seldom affects the current direction and hence maintains the balance of the sediment transport in the harbor. On the other hand, such a breakwater cannot stop strong currents and/or sediment transport, which might affect the operation of the harbor. The early permeable breakwater, which was proposed by Wiegel (1961), consisted of several rows of narrowly spaced piles and was gradually developed into a pile-foundation permeable breakwater with the upper part serving a wave dissipating function. In recent years, various permeable breakwaters were developed and applied in engineering projects, i.e., the horizontal plate breakwater (Patarapanich and Cheong, 1989; Nagata et al., 2003; Liu et al., 2008; Kee, 2009), T-type breakwater (Neelamani and Rajendran, 2002), suspended pipe breakwater (Mani and Jayakumar, 1995), and floating breakwaters (He et al., 2012). The horizontal twin-plate breakwater is probably the most effective one among these permeable breakwaters. It is well known that most of the wave energy concentrates near the water surface. The horizontal twin-plate breakwater consists of two horizontal plates, and the upper plate is placed near the water surface to dissipate the wave energy effectively. Besides, the breakwater has little effect on the propagation of the tide and current, which is preferable for natural coastal environments.

Received August 1, 2016; revised manuscript received by the editors December 5, 2016. The original version was submitted directly to the Journal.

KEY WORDS: Twin-plate breakwater, oblique random wave, wave dissipation, directional spectrum, model test.
The study of the horizontal plate breakwater started approximately 40 years ago. Siew and Hurley (1977) obtained the empirical formula for the transmission and reflection coefficients of the single submerged plate by using the matched asymptotic expansion method. Patarapanich and Cheong (1989) conducted experiments on the transmission and reflection coefficients of a submerged single plate and calculated the wave energy dissipation. Liu and Iskandarani (1991) solved the reflection and transmission coefficients of the single submerged horizontal plate with the eigenfunction method and concluded that the single plate could absorb energy well under long-period waves. With the increase in the water depth, however, the gap between the plate and seabed became wider, and most of the wave energy could transfer through the breakwater. As such, the dissipation performance decreased significantly and could not meet the expectation. To address this issue, researchers started to study the twin-plate and multi-plate breakwaters.

The reflection and transmission coefficients of a horizontal twin-plate system, which consists of a submerged leeward plate and a submerged seaward plate below the leeward plate, have been derived by Cheong and Patarapanich (1992). Their experimental results showed that the optimal submergence of the seaward plate was approximately $0.1-0.2$ to obtain the minimum wave transmission. Usha and Gayathri (2005) further performed a theoretical study of the wave reflection and transmission over a twin-plate breakwater with the velocity potentials in four fluid domains determined by three sets of orthogonal eigenfunction. In their study, the energy dissipation was neglected when the waves passed through the twin-plate system under the finite water depth. The optimal relative plate width and the submergence spacing for the minimum wave transmission were proposed. In addition, the study showed that the performance of the twin-plate breakwater is superior to that of the single floating and submerged plate or a group of submerged plates (e.g., three to six plates). Neelamani and Gayathri (2006) estimated the energy loss coefficient $k_{l}$ 